\title{
Reflectance reduction of InP wafers after high-temperature annealing
}

\author{
Oleg G. Semyonov, ${ }^{*}$ Arsen V. Subashiev, Alexander Shabalov, Nadia Lifshitz, \\ Zhichao Chen, Takashi Hosoda, and Serge Luryi \\ State University of New York at Stony Brook, Department of Electrical \\ and Computer Engineering, Stony Brook, New York 11794, USA \\ ${ }^{*}$ Corresponding author: osemyonov@ece.sunysb.edu \\ Received 15 March 2012; revised 3 May 2012; accepted 30 May 2012; \\ posted 3 July 2012 (Doc. ID 164909); published 26 July 2012
}

\begin{abstract}
Broadband reduction of light reflection from the surface of InP wafers after high-temperature annealing in air has been observed. In the transparency region of the material, the reflection drop is accompanied by increasing transmission of light through the wafer. The spectral position of a deep minimum of the reflection coefficient can be tuned, by varying the temperature and the time of annealing, in a wide spectral range from ultraviolet to infrared. The effect is due to formation of thermal oxide layers on the surfaces of the wafer with optical parameters favorable for antireflection. (C) 2012 Optical Society of America

OSIC codes: $160.6000,240.6490$.
\end{abstract}

\section{Introduction}

Suppression of the surface reflectance is beneficial for many devices, such as solar cells, LEDs, and sensors. Various methods of reflection reduction have been intensely studied, including multilayered coatings, nanoprofiling of the surface, and sputtering of metallic nanoparticles [1-4]. The traditional approach is to use the surface coating with a layer of transparent material having a lower refractive index than the index of the substrate or with a layer of a gradient refractive index. The reflectance reduction of a material can also occur due to modification of its surface layer. It is a common experience that the surface layers of semiconductor wafers are significantly modified in the process of high-temperature annealing. In particular, the annealing of InP results in depletion of phosphorous in the surface layers [5] and in the formation of an oxide layer of complex composition [6,7]. The reported refractive indices of InP oxides depend on the oxidation procedure. The indices of 1.49-1.55 in the visible range, measured

$1559-128 \mathrm{X} / 12 / 225425-07 \$ 15.00 / 0$

(C) 2012 Optical Society of America for the oxide grown electrochemically [요, 9$]$, are cited in the optical databases [10]. For thermal oxides $[6,7,11]$, the refractive index seems to be close to 1.9 . Either way, the refractive index of oxide layers is lower than that of the wafer material $n \approx 3.4$ near the absorption edge.

This paper studies the reflection and transmission of $n$-type InP wafers in the spectral range from 0.4 to $6 \mathrm{eV}$ after their annealing in air at different temperatures and for different times. Our earlier studies $[12,13]$ of optical properties of $n$-doped bulk InP showed high quantum efficiency of luminescence ( 95\%) which makes InP an effective scintillator for radiation detection. The surface reflectance reduction could be beneficial for luminescence extraction from the materials with a high refractive index.

\section{Experiment}

Two $350 \mu \mathrm{m}$ thick $n$-InP wafers [14] doped by sulfur with concentration $2 \times 10^{17} \mathrm{~cm}^{-3}$, both sides electrochemically polished, were cut into quarters. One quarter of each wafer was kept unprocessed as the reference sample. Three quarters of one wafer were annealed in an oven at 350,500 , and $600{ }^{\circ} \mathrm{C}$ for 30 min under atmospheric pressure, and three 
quarters of the other wafer were annealed at $600{ }^{\circ} \mathrm{C}$ for 30, 38, and $45 \mathrm{~min}$. The raw reflection $R_{\text {tot }}(E)$ and transmission $T_{\text {tot }}(E)$ spectra of all samples were scanned using a PerkinElmer Lambda-950 spectrophotometer in the wavelength range from $200 \mathrm{~nm}$ to $3 \mu \mathrm{m}$ (photon energy $6.22>E>0.414 \mathrm{eV}$ ). From the raw data for $R_{\text {tot }}(E)$ and $T_{\text {tot }}(E)$, the reflection coefficient $R(E)$ and the transmittance $t=$ $\exp [-\alpha(E) d]$ (here $d$ is the wafer thickness and $\alpha$ the absorption coefficient) were numerically calculated, accounting for the multiple reflections of the probe light beam from both sides of the samples, viz. [15-17]

$$
\begin{aligned}
& R_{\mathrm{tot}}=R\left(1+\frac{(1-R)^{2} t^{2}}{1-R^{2} t^{2}}\right), \\
& T_{\mathrm{tot}}=\frac{(1-R)^{2} t}{1-R^{2} t^{2}} .
\end{aligned}
$$

Equations (1) and (2) do not include corrections for the phase shift of the reflected wave in the region of high wafer absorption. For our thick samples, nontransparent in the absorption region, these corrections are estimated to be negligible. Equations (1) and (2) are derived for a single slab of reflecting and absorbing material. It was also shown [18] that the single-layer Eq. (1) and (2) are applicable to a symmetric multilayer system, so the InP slab with the oxide films on its sides can be treated as one effective slab with the surface reflection coefficients modified by the grown oxide films. Flipping the wafers upsidedown results in same raw curves; therefore, both surfaces are assumed to have the same reflection coefficient $R(E)$.

The raw reflection $R_{\text {tot }}$ and transmission $T_{\text {tot }}$ as well as the reflection coefficients $R(E)$ for the samples annealed at 350,500 , and $600{ }^{\circ} \mathrm{C}$ for $30 \mathrm{~min}$ are shown in Figs. 1(a) and 1(b). For the sample annealed at $350{ }^{\circ} \mathrm{C}$, the energy dependence $R(E)$ is close to that of the reference sample and InP reflectance spectrum from [10,19] Strong reflectance reduction in a broad energy range is observed at the annealing temperature $500{ }^{\circ} \mathrm{C}$ and higher. A deep minimum on the reflection graphs is observed for the anneal temperatures $500^{\circ} \mathrm{C}$ and $600{ }^{\circ} \mathrm{C}(30$ min annealing time $)$ with the minimum values of the reflection coefficient $R_{\min }=0.013$ and 0.002 for the photon energies
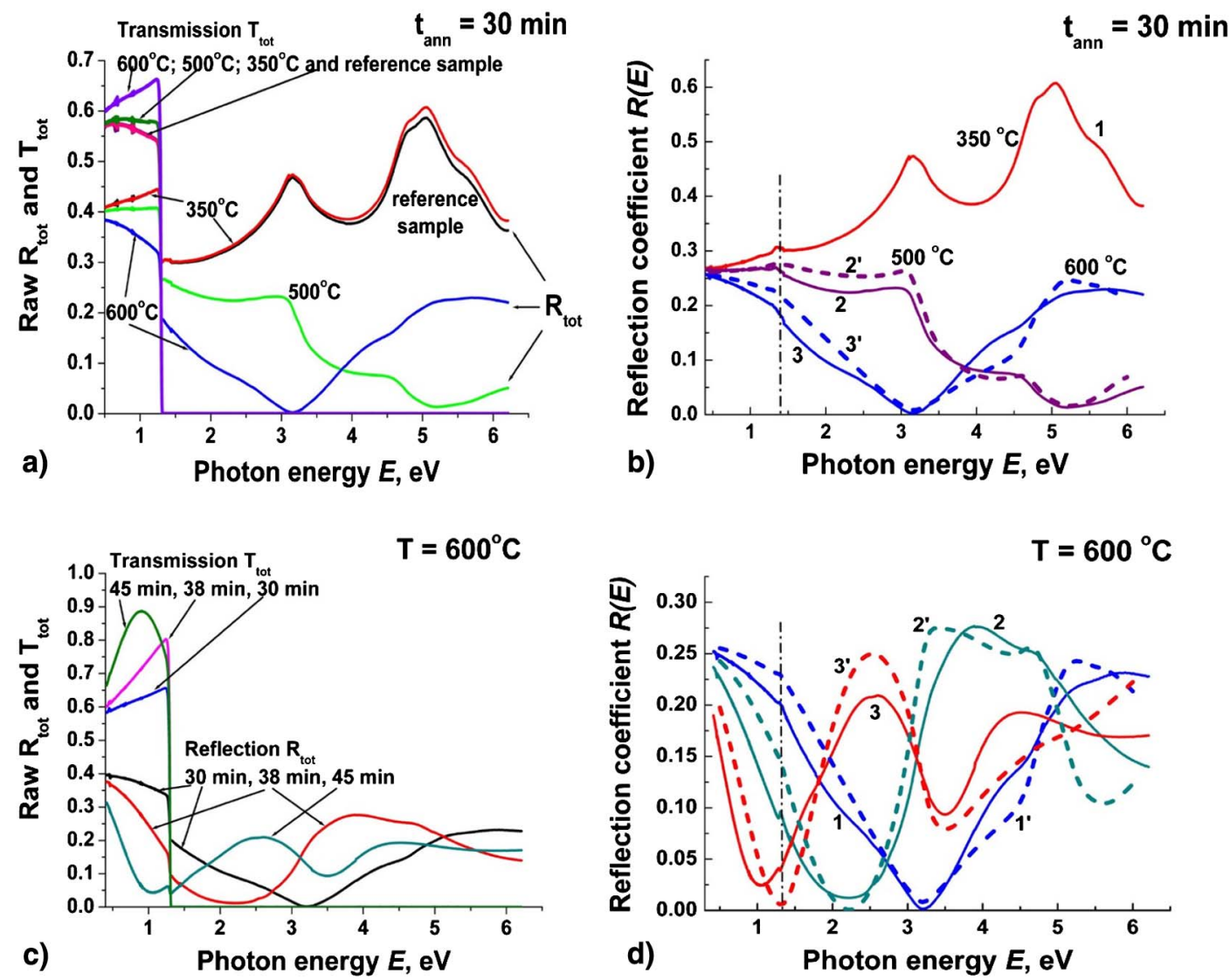

Fig. 1. (Color online) (a) Raw reflection $R_{\text {tot }}$ with raw transmission $T_{\text {tot }}$ and (b) reflection coefficient $R(E)$ (solid curves) as functions of photon energy for the samples annealed at 350 (1), $500(2)$, and $600{ }^{\circ} \mathrm{C}$ (3) for $t_{\text {ann }}=30$ min. (c) Raw reflection $R_{\text {tot }}(E)$ with raw transmission $T_{\text {tot }}(E)$ and (d) reflection coefficient $R(E)$ (solid curves) as functions of the photon energy for $\operatorname{InP}$ samples annealed at $T=600{ }^{\circ} \mathrm{C}$ during $t_{\text {ann }}=30$ (1), 38 (2), and $45 \mathrm{~min}$ (3). Also shown are the fits (dashed curves) of the experimental graphs $R(E)$ in the model of a homogeneous oxide layer (see details in the text). The sharp drop of transmission $T_{\text {tot }}$ in (a) and (c) and the vertical dash-dot lines in (b) and (d) indicate the position of the absorption edge of our InP samples. 
$E_{\min }=5.2 \mathrm{eV}$ and $3.19 \mathrm{eV}$, correspondingly. Figures 1(c) and 1(d) show the spectral dependence of $R_{\text {tot }}, \overline{T_{\text {tot }}}$, and the reflection coefficients $R(E)$ of the samples annealed at $T=600^{\circ} \mathrm{C}$ for 30,38 , and $45 \mathrm{~min}$. With the increasing annealing time, the minimum of reflection coefficient progressively shifts from ultraviolet to infrared (into the sample's transparency band), namely from $3.19 \mathrm{eV}\left(R_{\min }=0.002\right)$ for $30 \mathrm{~min}$ annealing to $1.14 \mathrm{eV}\left(R_{\min }=0.027\right)$ with 45 min annealing. In the transparency band, the reflection reduction is accompanied by a dramatically enhanced raw transmission with the calculated transmittance $t \approx 98.8 \%$. For the annealing times longer than $30 \mathrm{~min}$, an additional minimum appears in the ultraviolet, which corresponds, apparently, to the next interference minimum of an oxide layer grown on the surface. This minimum is spectrally shifted due to the dispersion both in the refractive indices and in the absorption coefficients of the wafer material and the layer itself.

To explore the surface of the samples after their high-temperature annealing, we performed the surface scans with scanning electron (SEM) microscope. SEM scans [Figs. 2(a) and 2(c)] of the samples annealed at $600{ }^{\circ} \mathrm{C}$ revealed patterning of the undersurface layers. The images of the samples' surfaces and the cleaved sides of the samples were obtained with an electron beam of 15 and $7.5 \mathrm{keV}$, respectively. The darker patches on the surface images correspond to the regions of relatively lower effective atomic weight (presumably $\mathrm{InPO}_{4}$ oxide clusters), while the irregular brighter network indicates the material of higher effective atomic weight. The SEM map of the surface for the sample annealed for $30 \mathrm{~min}$ at $T=600^{\circ} \mathrm{C}$ revealed the average lateral size of the darker irregularities $\sim 50-100 \mathrm{~nm}$. X-ray microanalysis of the samples' surface show no noticeable oxygen peak in the characteristic spectra of the reference sample and the sample annealed at $350{ }^{\circ} \mathrm{C}$ for $30 \mathrm{~min}$. For the samples annealed at $T=500^{\circ} \mathrm{C}$ and higher, the oxygen peak increases sharply indicating the substantial growth of an oxide layer [17].

The SEM images of the cleaved edges of the annealed wafers, shown in the bottom parts of the images in Fig. 2, also reveal the darker (presumably, oxide) clusters just beneath the surface [see also an insert in Fig. 2(a)], with their lateral sizes corresponding to the dark spots on the surface images and with their depth growing with the annealing time. No such patterns are observed in the reference samples. X-ray microanalysis of the cleaved edge cross section beneath the dark-spot layer showed no sign of oxygen while in the layer itself the oxygen content is high.

Earlier studies of thermal oxidation of $\operatorname{InP}$ in oxygen ambient, in particular, time-resolved massspectrometry of the surface layers [20] favored the InP oxidation model, according to which oxidation starts at the bare InP surface with the formation of $\mathrm{InPO}_{4}$ oxide. As $\mathrm{InPO}_{4}$ thickens, the diffusion flux of the atomic species through the oxide layer

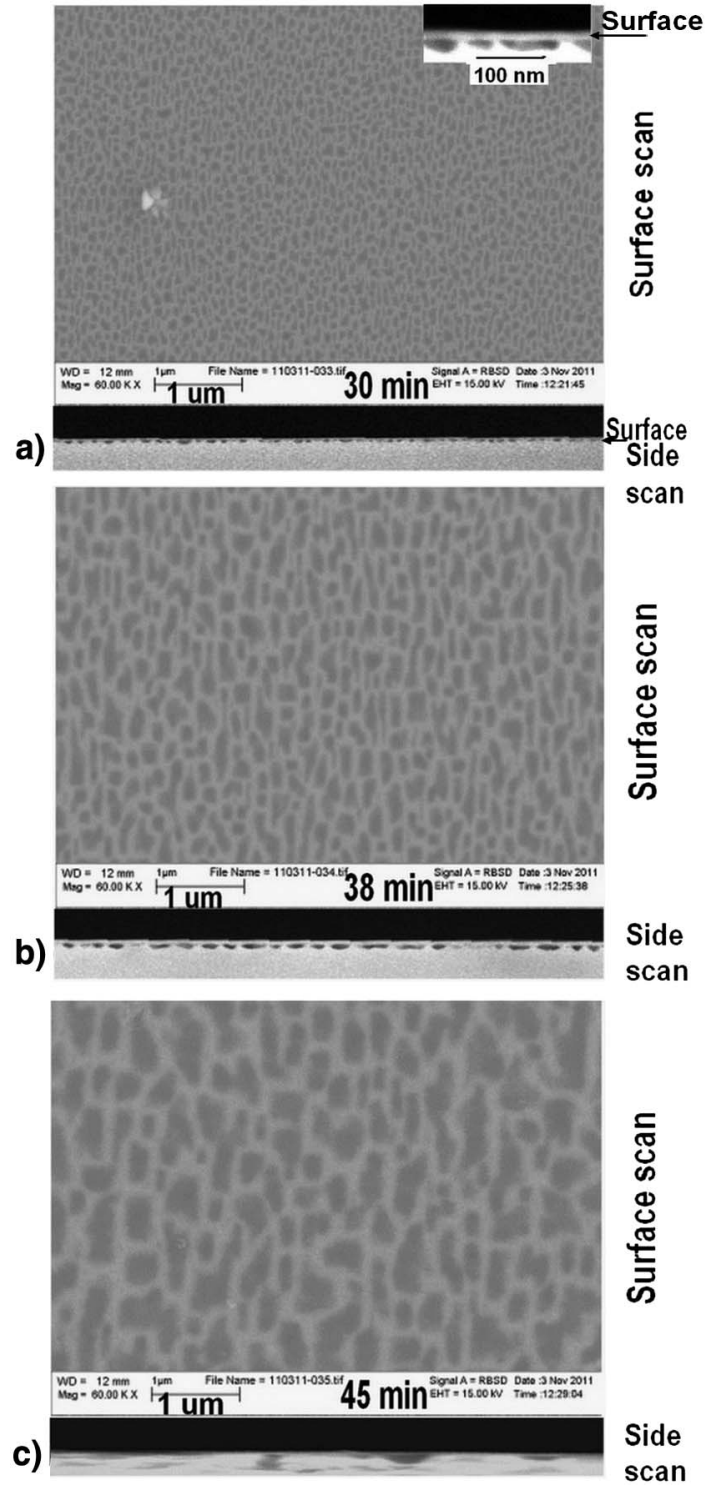

Fig. 2. SEM (backscattered electrons) images of the samples annealed at $600{ }^{\circ} \mathrm{C}$, for (a) 30, (b) 38, and (c) $45 \mathrm{~min}$, respectively. The darker areas correspond to the patterns of lower density in terms of the effective atomic weight (presumably oxide clusters). Note that their lateral size grows with the annealing time from 50 to $100 \mathrm{~nm}$ (a) to 0.5 to $1 \mu \mathrm{m}$ (c). The corresponding SEM images of the cleaved sides of the samples are shown at the bottom section of each micrograph. The magnified image of a small part of the side SEM image for $30 \mathrm{~min}$ annealing is also shown in the insert on (a).

decreases and the competitive processes become essential, in particular the decay of $\mathrm{InPO}_{4}$ into $\mathrm{In}_{2} \mathrm{O}_{3}$ and $\mathrm{P}_{2} \mathrm{O}_{5}$. The latter oxide tends to evaporate from the oxide surface leaving an In-enriched outer oxide layer, which may include the lower oxides like $\operatorname{In}_{4} \mathrm{O}_{2}$ and $\mathrm{In}_{2} \mathrm{O}$ [6]. As seen from the SEM images in Fig. 2 , the oxide formation differs from the model of laterally uniform $\mathrm{InPO}_{4}$ and In-enriched sublayers of the growing oxide layer assumed in [20]. Presumably, the oxidation starts in small local patches on the surface, where the lattice defects make oxidation easier, and then the formed $\mathrm{InPO}_{4}$ oxide clusters grow in size, with their effective atomic mass of $27 \mathrm{amu}$ being 
significantly smaller than the effective atomic mass of InP (72 amu). The clusters are distinguishable as the relatively darker regions beneath the oxide surface growing with temperature and annealing time (compare the side SEM images for 30 and $38 \mathrm{~min}$ anneals), while the In-enriched crust (presumably a mixture of $\operatorname{In}_{2} \mathrm{O}_{3}$ and lower oxides with the effective atomic weight exceeding $56 \mathrm{amu}$ ) are formed on their outer surfaces (a brighter-looking layer on top of them), as clearly seen on the insert in Fig. 2(a) and on the side SEM image in Fig. 2(b). Remarkably, the surface relief of the wafers obtained with very lowenergy $(2.5 \mathrm{keV})$ short-penetration electrons does not reveal this patterning, and the typical sizes of the surface relief are $\sim 10-20 \mathrm{~nm}$ in height and about $100 \mathrm{~nm}$ laterally. It means the oxidation penetrates into InP and results in a growing depth of the oxide clusters, while the surface relief is subjected to relatively minor changes. This can be also seen from the images of the cleaved edges of the samples in Fig. 2.

The earlier performed atomic force microscope (AFM) scan of the samples' surfaces [17] showed some modification of the surface morphology after annealing at temperatures $500{ }^{\circ} \mathrm{C}$ and higher, while the reference and the $350{ }^{\circ} \mathrm{C}$ annealed samples display flat surface areas with irregular elevations apparently related to dust granules on the surface. A thicket of "hills" with their lateral size $\sim 50-100 \mathrm{~nm}$ and with the average height increasing with the annealing temperature from 5 to $10 \mathrm{~nm}$ for $500{ }^{\circ} \mathrm{C}$ to 10 to $17 \mathrm{~nm}$ for $600{ }^{\circ} \mathrm{C}(30 \mathrm{~min})$ is formed over the whole surface at higher annealing temperatures. The average lateral size of the "hills" $(\sim 100 \mathrm{~nm})$ seems to grow marginally with the annealing time.

\section{Discussion}

The reflection and transmission spectra were analyzed with different assumptions about the surface structure of the oxide layer. The notable feature of the experimental spectra is the almost-complete suppression of reflection at particular photon energy and the shift of the spectral position of the reflection minimum to lower photon energies with increasing temperatures and annealing times. We have found that a homogeneous oxide-layer model can reproduce these features. In order to get nearly total antireflection, two conditions must be met: (i) The phase shifts for light waves reflected by different interfaces must be matched; and (ii) the index of refraction $n$ of the layer must be close to the square root $\left(n_{s}\right)^{1 / 2}$ of the substrate refractive index. To calculate the reflection spectra $R(E)$ for a homogeneous oxide layer on the InP surface, we used the conventional single-layer equation for the reflection from an oxide layer on the InP wafer with the energy dependent complex refractive index $n+i \kappa$ of $\operatorname{InP}$ [19] in combination with Eqs. (1) and (2). Our calculations showed that the antireflection condition (ii) is rather relaxed: An oxide layer with the index in the range 1.75 to 2 and a thickness above $20 \mathrm{~nm}$ will lead to a reflection minimum with $R_{\min } \leq 0.2 \%$. The strong variation of $n$ and $\kappa$ of InP with the photon energy narrows the range of suitable refractive indices for the oxide. Thus, the database [10] value $(n \approx 1.4)$ is too low for the observed nearly total antireflection.

The results of our calculations for the samples annealed at $600{ }^{\circ} \mathrm{C}$ are shown in Fig. 1(d). For the annealing times of 30,38 , and $45 \mathrm{~min}$ at $600{ }^{\circ} \mathrm{C}$, the fitting (dotted curves $1^{\prime}, 2^{\prime}$, and $3^{\prime}$ ) in the model of homogeneous oxide layer gives the approximate thicknesses $d=44,68$, and $122 \mathrm{~nm}$, respectively. The closest fit was obtained with both the real part $n$ and the imaginary part $\kappa$ of the refractive index dependent on the photon energy: $n(E)=1.95+1.4 \times$ $10^{-3} E^{2}+4 \times 10^{-5} E^{4}$ and $\kappa(E)=2.8 \times 10^{-2}(E-1.5)^{2}$, where $E$ is in eV. Our values of $n$ are larger than 1.6 quoted by Studna and Gualtieri [8] but close to 1.9 cited by Nelson et al. [7]. These dependences $n(E)$ are reasonably close to those measured by Robach et al. [11], where $n(E)$ ranged from 1.85 to 2 . To fit the reflection curve for the sample with the annealing time of $45 \mathrm{~min}$, the index $\kappa$ is taken as $\kappa=3.6 \times 10^{-2}(E-0.5)^{2}$, which corresponds to the higher oxide layer absorption in this sample. In the reflection spectra of Fig. 1(d), the higher $\kappa$ is manifested by lowered reflection at the interference maxima of the reflection spectra and significantly enhanced reflection in the interference minima above $3 \mathrm{eV}$. Quadratic growth of absorption is typical for materials with multiple structural defects or amorphous materials [21]. The increasing thickness of the oxide layer composed of a disordered mixture of various clustered oxides is, apparently, accompanied by the enhanced absorption and light scattering. The layer is thick enough to provide a strong antireflection effect when its optical thickness is close to $\lambda / 4$ (deviations of the spectral positions of the observed interference minimums in the high-energy spectral band [from their positions estimated with $n d=\lambda / 4$ for the complex refractive index $n(E)$ above] can be linked to the growing absorption of the InP substrate with photon energy, which causes an additional phase shift). The higher experimental value of refractive index of the oxide layer compared to $\mathrm{In}_{2} \mathrm{O}_{3}$ oxide may be due to inclusions of the dielectric compounds $\operatorname{InPO} \mathrm{P}_{4}$ and $\operatorname{In}\left(\mathrm{PO}_{3}\right)_{3}[7,19]$.

We examined [17] other models and assumptions about the structure of the oxide layer and found that they do not agree with the experimental curves $R(E)$ over the entire range of photon energies. The Bedeaux model [22], based on the assumption of a thin surface layer with its polarizability expanded in multipole moments, can fit well the experimental curves $R(E)$ in the long-wavelength region $(E<1.5 \mathrm{eV})$. However, it fails to reproduce the $R(E)$ features including the second interference minimum of the reflection coefficient at higher energies. The same can be said about the plasmonic antireflection, if one assumes a random set of metallic In nano-droplets suspended in the oxide layer. If, based on the SEM 
images, the modified layer is interpreted as $\operatorname{In}_{2} \mathrm{O}_{3}$ oxide clusters mixed with InP inclusions [brighter mesh in Fig. 2(a)-2(c)], the layer dielectric function should be an average of the dielectric functions of the oxide and $\operatorname{InP},\langle\varepsilon\rangle=\varepsilon_{\operatorname{InP}} x+\varepsilon_{\text {ox }}(1-x)$, where $x$ is the volume content of InP in the layer. The latter model could produce an enhanced oxide index observed experimentally; however, the average $\langle\varepsilon\rangle$ would retain some of the characteristic features of $\varepsilon_{\text {InP }}$ and result in a markedly different spectral behavior of the reflection coefficient [17].

The lower annealing temperatures result in reduced oxidation rates and hence thinner oxide layers formed during a fixed annealing time. The experimental reflection spectra, for two anneal temperatures of 500 and $600{ }^{\circ} \mathrm{C}$ and the same annealing time of $30 \mathrm{~min}$, are shown in Fig. 1(b). For $T=$ $500{ }^{\circ} \mathrm{C}$, the reflection spectrum has a characteristic structure inherited from the refractive index variation of InP with a deep antireflection minimum near $E=5.2 \mathrm{eV}$. The thickness of the oxide layer estimated from the condition $d \approx \lambda / 4 n$ is $28 \mathrm{~nm}$ for $n=2$. More accurate calculation allowing for the substrate absorption gives $d=22 \mathrm{~nm}$. At $T=$ $600^{\circ} \mathrm{C}$, the reflection minimum shifts to $E=3.2 \mathrm{eV}$, which corresponds to thickness $d \approx 44 \mathrm{~nm}$. The reflection spectra calculated in the model of a homogeneous oxide layer are shown in Fig. 1(b) by the dashed lines. The model also gives an excellent account to our results for $500{ }^{\circ} \mathrm{C}$ annealing temperature. The best fit is obtained with a slightly lower absorption index of the oxide layer, viz. $\kappa=1.7 \times$ $10^{-2}(E-1.5)^{2}$ and a correspondingly slightly smaller dispersion of the real index at high energies, $n=1.95+1.4 \times 10^{-3} E^{2}+1.5 \times 10^{-5} E^{4}$.

To check the validity of the quasihomogeneous layer model we have measured the transmission of a $980 \mathrm{~nm}$ laser beam (in the transparency region of InP) as a function of the angle of incidence separately for $s$ - and $p$-polarization both for the reference sample and for the sample annealed at $T=600^{\circ} \mathrm{C}$ during $45 \mathrm{~min}$. For the reference sample, the total transmission of $p$-polarized light grows with the angle of incidence from $T_{\text {tot }}=0.51$ toward a maximum $T_{\text {tot }} \approx 0.94$ near the Brewster angle at $\varphi \approx 73^{\circ}$ which corresponds to the refractive index of $\operatorname{InP} n=3.36$, while the transmission of $s$-polarized light uniformly declines with the angle of incidence. For the sample annealed at $600{ }^{\circ} \mathrm{C}$ for $45 \mathrm{~min}$, the transmission of $p$-polarized light increases slowly from $T_{\text {tot }}=0.8$ toward $T_{\text {tot }} \approx 0.86$ at a pseudo-Brewster angle $\varphi \approx 55^{\circ}$.

The homogeneous layer model gives a good fit to the angular dependence of the total transmission in the transparency region, as shown in Fig. 3 by the solid lines. It should be noted that the angular dependence of $p$-polarized wave is sensitive not only to the effective optical thickness $n d$, but to the index $n$ and the thickness $d$ of the interlayer individually. An excellent agreement with the experimental results was obtained for $d=100 \mathrm{~nm}$ and $n=1.95$. The refractive index is identical to that estimated earlier from

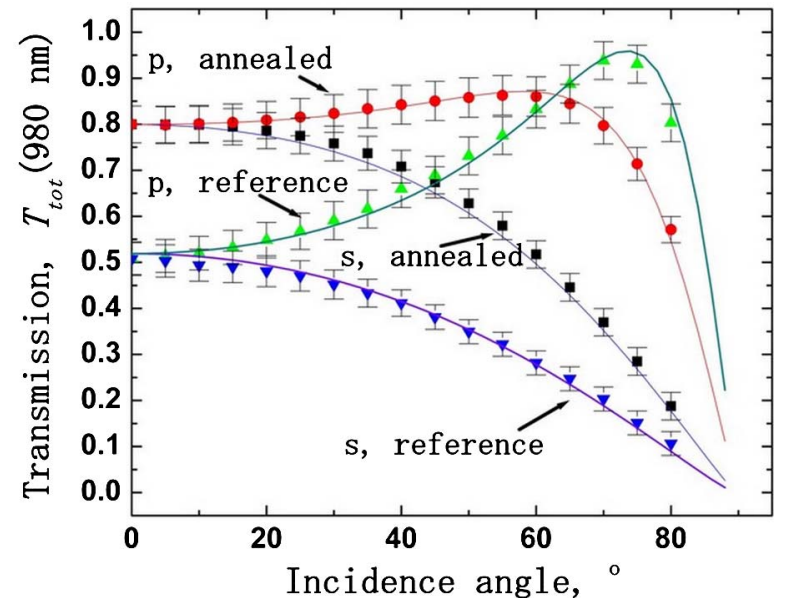

Fig. 3. (Color online) Experimental transmission $T_{\text {tot }}$ of an original (reference) sample and the sample annealed at $T=600^{\circ} \mathrm{C}$ for $45 \mathrm{~min}$ (dots) as a function of the incidence angle for $s$ - and $p$ polarizations of the incident laser beam of $980 \mathrm{~nm}(E=1.267 \mathrm{eV})$ (dots). Solid lines show the calculated data (see the text).

the experimental reflection spectrum in the wide energy range. On the other hand, the thickness $d$ is somewhat smaller than the $122 \mathrm{~nm}$ estimated earlier.

Using the calculated $n(E)$ and $\kappa(E)$, one can find the energy dependence of the complex dielectric function $\varepsilon$ of the surface oxide layer. Its real and imaginary parts, $\operatorname{Re} \varepsilon$ and $\operatorname{Im} \varepsilon$, are shown in Fig. 4 for the annealing temperature $T=600^{\circ} \mathrm{C}$. Also shown in Fig. 4 are the data for the thermal oxide layer from Robach et al. [11]. Both our results and those of [11] are obtained by solving an inversion problem, which has a limited accuracy. While the absolute values for the real part of the dielectric function are reasonably close, the data [11] show significantly larger variations in $\operatorname{Re} \varepsilon$ together with a steep decrease of $\operatorname{Re} \varepsilon$ in the high-energy region, where $\operatorname{Im} \varepsilon$ continues to grow. This is hard to reconcile with the KramersKronig relation, unless one postulates the existence of a peak in absorption just outside the energy range

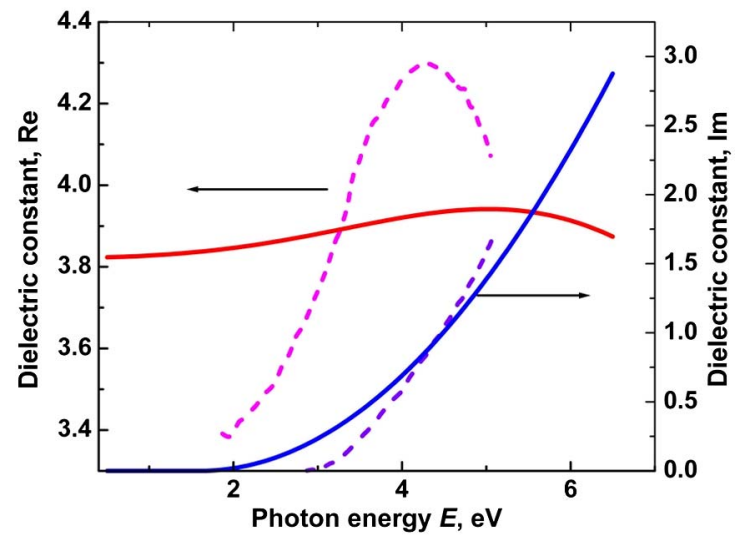

Fig. 4. (Color online) Dependence of real and imaginary parts of dielectric function of the oxide layer evaluated from fitting the interference pattern of the reflection spectra at $600{ }^{\circ} \mathrm{C}$. Dashed curves show $\operatorname{Re} \varepsilon$ and $\operatorname{Im} \varepsilon$ according to Robach et al. [11]. 
of our experiment. Within our range this would imply a strong increase of absorption with energy, much stronger than the observed quadratic increase. Our data for $\operatorname{Im} \varepsilon$ are close to those of [11], but our quadratic energy dependence is "theoretically motivated" and better suited for different annealing conditions.

It may look surprising that a model of a homogeneous oxide layer works so well in a structure with the oxide layers that are manifestly nonuniform in terms of the effective atomic weight; cf. Fig. 2. Possibly, the geometry of reflectance measurements with a relatively wide probe beam imposes a self-averaging effect over a large area on the layer surface, so that an average index of the surface layer is measured. The main manifestation of the optical inhomogeneity is light scattering. Virtually no scattered laser light (only specular reflection) is visible in the infrared $(980 \mathrm{~nm})$ from the sample annealed for $30 \mathrm{~min}$ at $600{ }^{\circ} \mathrm{C}$ and from the samples annealed at lower temperatures. The increase of the lateral size of these spots from 50 to $100 \mathrm{~nm}$ for $30 \mathrm{~min}$ annealing at $T=600{ }^{\circ} \mathrm{C}$ to 0.5 to $1 \mu \mathrm{m}$ for $45 \mathrm{~min}$ annealing time can explain the appearance of some scattered laser light observed with an infrared viewer from the surface of the sample annealed for $45 \mathrm{~min}$ at $600{ }^{\circ} \mathrm{C}$ irradiated by a $980 \mathrm{~nm}$ laser beam, for which the size of the pattern in Fig. 2 become comparable with the laser wavelength, though the intensity of the scattered light was rather small. It should be noted that the surface relief of the oxide layer has a significantly smaller spatial scale than the wavelength of photons in the transparency region of InP; hence it produces negligible scattering of infrared light. Closer to the ultraviolet spectral band, both the relief and the variations of refractive index between the lower atomic weight inclusions and the higher atomic weight regions in the oxide layer can be responsible for higher light scattering.

Note that the refractive index and the real part of dielectric function do not vary for annealing temperatures below $600{ }^{\circ} \mathrm{C}$. This means that we can control the position of the reflectance minimum by timing the annealing at temperatures where the electrical and luminescence properties are stable (below $620{ }^{\circ} \mathrm{C}[23]$ ).

The described antireflection effect associated with surface oxidation is not exclusive to InP but is also observed with other semiconductors. In particular, we observed the anneal-induced antireflection effects in GaAs and GaP wafers [17]. For GaP samples, the substantial reflection reduction effect appears at the annealing temperature of $750^{\circ} \mathrm{C}$ (no changes at $600^{\circ}$ $\mathrm{C}$ and below) and the minimum of reflection also shifts to lower energies with the increasing annealing time. For GaAs, the antireflection is observed at $600{ }^{\circ} \mathrm{C}$ after 30 min anneal but in contrast with InP and $\mathrm{GaP}$, due to the different oxidation kinetics, the deep $(R<3 \%)$ reflection minimum shows virtually no red shift with the excess annealing times above 30 min. The higher index $(n \approx 1.9)$ of GaAs oxide, compared with $n \approx 1.6$ for GaP oxide [10] results in a much deeper antireflection effect [17].

\section{Conclusion}

Reflectance reduction of InP samples upon their high-temperature annealing in air has been observed. In all cases, we find a deep minimum in the reflection spectrum. The position of the antireflection minimum is shifted from the UV to the IR region with the increase of anneal temperature (above $500{ }^{\circ} \mathrm{C}$ ) and/or time (above $30 \mathrm{~min}$ ). The AFM and SEM microscopy showed that roughness and patterning of the surface layer grow with the annealing temperature and the time of annealing, accompanied by the overall increase of the effective thickness of the layer with time. The thickness and optical homogeneity of the surface layer was estimated by fitting experimental spectra of the reflection coefficient with calculations based on different models of the oxide layer. The closest fit to the experimental spectra in a broad energy range from 0.4 to $6 \mathrm{eV}$ was obtained with the model of an optically homogeneous oxide layer having an almost constant real part $\operatorname{Re} \varepsilon \approx 3.9$ and the imaginary part $\operatorname{Im} \varepsilon$ increasing with photon energy as in quasi-amorphous materials.

We found that the index of thermal oxide on $\mathrm{InP}$ is considerably higher than that cited in most databases but is in agreement with some earlier studies of thermal oxidation. The high oxide index gives rise to a deep anti-reflection minimum. Position of the deep minimum can be tuned in a wide range by varying the effective thickness of the oxide layer controlled by the anneal time and/or temperature.

We note that the substantial reduction of reflection is also observed on the surfaces of other $A_{3} B_{5}$ semiconductors after high-temperature annealing. The approach used here can be applied to obtain more detailed information about the structure of the modified surface layers.

This work was supported by the Department of Homeland Security through its Academic Research Initiative, by the Defense Threat Reduction Agency through its Basic Research program, and by the NY State Center for Advanced Sensor Technology at Stony Brook.

\section{References and Notes}

1. S. Chhajed, M. F. Schubert, J. K. Kim, and E. F. Schubert, "Nanostructured multilayer graded-index antireflection coating for Si solar cells with broadband and omnidirectional characteristics," Appl. Phys. Lett. 93, 251108 (2008).

2. B.-J. Kim and J. Kim, "Fabrication of GaAs subwavelength structure (SWS) for solar cell applications," Opt. Express 19, A326-A330 (2011).

3. S. Wang, X. Z. Yu, and H. T. Fan, "Simple lithographic approach for subwavelength structure antireflection," Appl. Phys. Lett. 91, 061105 (2007).

4. A. A. Cruz-Cabrera, L. I. Basilio, D. W. Peters, J. R. Wendt, S. A. Kemme, and S. Samora, "Fabrication and testing of plasmonic optimized transmission and reflection coatings," Proc. SPIE 6883, 68830R (2008).

5. J. D. Oberstar, B. G. Streetman, J. E. Baker, N. L. Finnegan, E. A. Sammann, and P. Williams, "Annealing encapsulants for 
InP I: Auger electron and secondary ion mass spectrometric studies," Thin Solid Films 94, 149-159 (1982).

6. M. Yamaguchi and K. Ando, "Thermal oxidation of InP and properties of oxide film,” J. Appl. Phys. 51, 5007-5012 (1980).

7. A. Nelson, K. Geib, and C. W. Wilmsen, "Composition and structure of thermal oxides of indium phosphide," J. Appl. Phys. 54, 4134-4140 (1983).

8. A. A. Studna and G. J. Gualtieri, "Optical properties and water absorption of anodically grown native oxide on InP," Appl. Phys. Lett. 39, 965 (1981).

9. W. F. Wu and B. S. Chiou, "Effect of annealing on electrical and optical properties of RF magnetron sputtered indium tin oxide films," Appl. Surf. Sci. 68, 497-504 (1993).

10. FreeSnell: Refractive Index Spectra nk-data, http://people .csail.mit.edu/jaffer/FreeSnell/nk.html; New Semiconductor Materials. Characteristics and Properties, $n, k$ database, http://www.ioffe.ru/SVA/NSM/nk/index.html.

11. Y. Robach, A. Gagnair, J. Joseph, E. Bergignat, and G. Hollinger, "Optical properties of native oxides on InP," Thin Solid Films 162, 81-88 (1988).

12. O. Semyonov, A. Subashiev, Z. Chen, and S. Luryi, "Radiation efficiency of heavily doped bulk $n$-InP semiconductor," J. Appl. Phys. 108, 013101 (2010).

13. S. Luryi and A. Subashiev, "Semiconductor scintillator for three-dimensional array of radiation detectors," in Future Trends in Microelectronics: From Nanophotonics to Sensors to Energy (Wiley Interscience, 2010), pp. 331-346.
14. We used ACROTEC wafers from NIKKO Metals.

15. S. M. Sze and K. K. Ng, Physics of Semiconductor Devices (Wiley, 2007), p. 54.

16. C. R. Bull, "A model of reflection of near-infrared radiation," J. Mod. Opt. 37, 1955-1964 (1990).

17. O. Semyonov, A. Subashiev, A. Shabalov, N. Lifshitz, Z. Chen, T. Hosoda, and S. Luryi, "Reduction of optical reflection from InP semiconductor wafers after high-temperature annealing," submitted to J. Appl. Phys., http://arxiv.org/abs/1112.5398v1 (2011).

18. S. D. Smith, "Design of multilayer filters by considering two effective interfaces," J. Opt. Soc. Am. A 48, 43-49(1958).

19. D. E. Aspnes and A. A. Studina, "Dielectric functions and optical parameters of $\mathrm{Si}, \mathrm{Ge}, \mathrm{GaP}, \mathrm{GaAs}, \mathrm{GaSb}, \mathrm{InP}, \mathrm{InAs}$ and In Sb from 1.5 to 6.0 eV," Phys. Rev. B 27, 985-1009 (1983).

20. X. Lui, M. S. Denker, and E. A. Irene, "An oxygen tracer study of InP oxidation," J. Electrochem. Soc. 139, 799-802 (1992).

21. J. Tauc, "Optical properties of amorphous semiconductors," in Amorphous and Liquid Semiconductors, J. Tauc, ed. (Plenum, 1974), p. 159.

22. D. Bedeaux and J. Vlieger, Optical Properties of Surfaces (Imperial College Press, 2004).

23. T. Inoue, H. Shimakura, K. Kainosho, R. Hirano, and O. Oda, "Annealing of undoped InP and evaluation by luminescence," J. Electrochem. Soc. 137, 1283-1288 (1990). 